\title{
Rib 5
}

National Cancer Institute

\section{Source}

National Cancer Institute. Rib 5. NCI Thesaurus. Code C52763.

The fifth rib counting from the top of the rib cage down. 